\title{
Respiratory Syncytial Virus NS1 Protein Degrades STAT2 by Inducing SOCS1 Expression
}

\author{
Xiaodong $\mathrm{Xu}^{\mathrm{a}, \mathrm{b}}$ Junwen Zheng ${ }^{\mathrm{a}}$ Kun Zheng ${ }^{\mathrm{a}}$ Yan Hou ${ }^{\mathrm{a}}$ Feng Zhao ${ }^{\mathrm{b}}$ \\ Dongchi Zhao ${ }^{a}$ \\ a Department of Pediatrics, Zhongnan Hospital of Wuhan University, Wuhan, and bepartment of Pediatrics, \\ Taihe Hospital Affiliated to Hubei University of Medicine, Hubei, China
}

\section{Key Words}

Respiratory syncytial virus · Nonstructural protein NS1 .

Suppressor of cytokine signaling $1 \cdot$ Signal transducer and activator of transcription $2 \cdot$ RIG-I mRNA - TLR3 mRNA

\section{Abstract}

Objectives: Respiratory syncytial virus (RSV) nonstructural protein NS1 (NS1) has been shown to block interferon (IFN)inducible antiviral signaling. The suppressor of cytokine signaling (SOCS) gene family could utilize a feedback loop to block the activation of the JAK/STAT signaling pathway, further inhibiting the activation of host type I IFN. We evaluated the role of the SOCS1 and SOCS3 genes in this antiviral mechanism. Material and Methods: A humanized stable NS1 (rich in GC)-expressing plasmid was constructed, and A549 cells were transfected with it. Expression of the SOCS1, SOCS3, RIG-I, and TLR3 mRNAs was measured with real-time PCR. STAT2 and PSTAT2 expression was determined with Western blotting. Results: RSV NS1 upregulated SOCS1 mRNA expression 30-fold increase compared with the baseline level in very early phase $(p<0.01)$, and silence of RIG-I or TLR3 mRNA did not affect NS1-induced SOCS1 expression. NS1 inhibited IFN-a-induced STAT2 phosphorylation and degraded STAT2 in a time-dependent manner compared

\section{KARGER}

(c) 2014 S. Karger AG, Basel

0300-5526/14/0572-0065\$39.50/0

E-Mail karger@karger.com

www.karger.com/int with the empty-vector control ( $p<0.05)$. Conclusion: RSV NS1 upregulates SOCS1 expression in a RIG-I- and TLR3-independent pathway, to inhibit STAT2 phosphorylation.

(C) 2014 S. Karger AG, Basel

\section{Introduction}

Respiratory syncytial virus (RSV) is the predominant pathogen involved in respiratory tract infections in infants and young children worldwide, and is closely associated with the development of allergic asthma later in life $[1,2]$. Despite its worldwide importance and several decades of research, there is still no vaccine or specific antiviral therapy for RSV disease [3]. RSV is an enveloped, nonsegmented, single-stranded, negative-sense RNA virus belonging to the subfamily Pneumovirinae of the family Paramyxoviridae [4]. The RSV genome encodes 11 proteins, including nonstructural (NS) proteins NS1 and NS2, which are the most important molecules mediating the virus's evasion of the host's immune surveillance [5, 6]. In the initial phase of viral replication, the NS1 gene,

X. Xu and J. Zheng contributed equally to this work as co-first authors. 
located at the $3^{\prime}$-endpoint of the RSV genome, is transcribed first, and the protein is synthesized in the cytoplasm to resist host antiviral reaction [5-8].

In vitro, RSV induces a lower level of interferon (IFN) and interferes with type I IFN signaling by degrading the signal transducer and activator of transcription (STAT) 2 protein and inhibiting STAT2 phosphorylation [9-12]. Recombinant RSV mutants lacking NS1 and NS2 cause marked production of host IFN- $\alpha$ and $-\beta$, highlighting the importance of these proteins in resisting the innate antiviral response $[7,8]$. It has been shown that the RSV NS1 protein degrades STAT2 by forming a functional E3 ligase which acts as suppressor of cytokine signaling (SOCS) proteins [12]. The SOCS family comprises eight proteins, of which SOCS1 and SOCS3 appear to be the most effective in regulating type I IFN expression $[13,14]$. The cytokine-activated JAK/STAT signaling pathway is blocked by SOCS proteins via a negative feedback regulatory mechanism [15-17]. SOCS binds to JAK and inhibits the activity of its catalytic kinase reaction to block the JAK/STAT signal transduction pathway, to inhibit STAT phosphorylation [17-19], and to interact with elongin B via the SOCS box, forming new complexes in the role of cullin-2 and the proteasome [12].

Virus infection upregulates the expression of the SOCS genes in a variety of ways. One way is mediated by the JAK/STAT signaling pathway induced by the secretion of endocrine cytokines, such as type I IFN [20]. Singlestranded RNA viruses and its duplication pattern of the intermediate double-stranded RNA can induce IFN transcription by activating the RIG-I and TLR3 pathways respectively, and the expression of the SOCS is upregulated by the production of endogenous IFN $[21,22]$. SOCS transcription can also be directly induced by the RSV surface proteins during viral replication [22]. The recombinant deletion mutant $\triangle \mathrm{NS} 1 / 2$ of RSV showed significantly reduced expression of SOCS1 and induced more IFN production than wild-type RSV [23]. These results indicate that other pathways also activate SOCS expression, independently of IFN signaling. In the present study, we hypothesized that RSV NS1 upregulates SOCS in an IFNindependent manner, and sought to confirm that NS1 protein directly induces SOCS1 expression.

\section{Materials and Methods}

\section{Cells and Virus}

Human alveolar type II-like epithelial cells, A549, were provided by the American Type Culture Collection the American Type Culture Collection (ATCC, Rockville, Md., USA) and were maintained in $75-\mathrm{cm}^{2}$ cell-culture flasks with Dulbecco's modified Eagle's medium containing $10 \%$ fetal bovine serum (with $100 \mathrm{U}$ of penicillin, $100 \mathrm{U}$ of streptomycin per $\mathrm{ml}$ ) at $37^{\circ}$ in a $5 \% \mathrm{CO}_{2}$ incubator. The cells were passaged when they had grown to a dense monolayer. RSV A2 strain was obtained from the ATCC, propagated and purified as previously described [25], and snap frozen until use. The viral titer was determined with a plaque titration assay in A549 cells.

\section{Plasmid Construction and Transfection}

To evaluate the regulatory effects of NS1 on SOCS1 and SOCS3, an NS1 expression plasmid was constructed with reference to the open reading frames (ORFs) of the RSV A2 strain. Because the NS1 sequence is rich in AT bases, the mRNA and protein expression levels are insufficient for analysis in host cells. Therefore, we constructed a plasmid (pNS1) to express a stable 'humanized' NS1 (rich in GC bases) keeping the original genetic information (GenBank accession, JQ900253). The amino acid sequences of the new NS1 ORFs were unaffected. A flag-tag sequence was added to the $3^{\prime}$ NS1 tail in the ORF by annealing an oligonucleotide bearing the flag-tag. The correct insertion of the flag-tag was determined by automated DNA sequencing. The plasmid was transiently transfected with cationic liposomes (Lipofectamine 2000; Invitrogen Corp., Carlsbad, Calif., USA), according to the manufacturer's instructions. When the cells reached $60 \%$ confluence, they were transfected with pNS1. For a 12-well plate, a mixture of $1.6 \mu \mathrm{g}$ of plasmid and $4 \mu \mathrm{l}$ of Lipofectamine 2000 was incubated in $200 \mu \mathrm{l}$ of Opti-MEM I Reduced Serum Medium (Opti-MEM; Gibco, Grand Island, N.Y., USA) at room temperature for $20 \mathrm{~min}$. The complexes were then added to each well containing cells and medium, without antibiotics. For 6-well plates, $4 \mu \mathrm{g}$ of plasmid and $10 \mu \mathrm{l}$ of Lipofectamine 2000 complex were transfected in each well. The final plasmid concentration was $8 \mu \mathrm{g} / \mathrm{ml}$. The transfected cells were placed in a $5 \% \mathrm{CO}_{2}$ incubator at $37^{\circ}$ for the indicated times.

\section{RNA Interference and Transfection}

Small interference RNAs (siRNAs) targeting SOCS1 and RSV NS1 mRNAs were designed and synthesized by Invitrogen Corp. The siRNA sequences were: siNS1 gene $5^{\prime}$-GUGAUUCAA CAAUGACCAAdTdT-3'; siSOCS1 gene 5'-CGCCGUGCACG CAGCAUUAAC-3' [11]; siTLR3 5'- GCUCGAUCUUUCCUA CAACAAdTdT-3'; siRIG-I 5' - GCAAGAUCUUACUCAGAGA UUdTdT- $3^{\prime}$. The siRNA inhibition efficiency was determined with real-time polymerase chain reaction (PCR). Once the cells had grown to $60 \%$ confluence, they were transfected with the siRNA to silence the corresponding mRNA. For 12-well plates, the siRNA and $2 \mu \mathrm{l}$ of Lipofectamine 2000 were incubated in $200 \mu \mathrm{l}$ of OptiMEM I Reduced Serum Medium at room temperature for $20 \mathrm{~min}$. The complexes were then added to each well of cells with medium lacking antibiotics. For 6-well plates, the siRNA and $5 \mu \mathrm{l}$ of Lipofectamine 2000 were incubated in $500 \mu \mathrm{l}$ of Opti-MEM I Reduced Serum Medium. The final concentration of siRNA was $40 \mathrm{~nm}$ in all experiments. The transfected cells were placed in a $5 \% \mathrm{CO}_{2}$ incubator at $37^{\circ}$ for $24 \mathrm{~h}$ until RSV infection or plasmid transfection.

RNA Reverse Transcription and Real-Time PCR

Total RNA was extracted according to the TRIzol Reagent manual (Tri Reagent; Invitrogen Corp.), and $1 \mu \mathrm{g}$ of RNA was reverse transcribed in a $20-\mu l$ reaction mixture using the M-MLV reverse transcription kit (Invitrogen Corp.). The SYBR Green PCR Master Mixture (Invitrogen Corp.) was used to perform the real-time PCR. 
The $25 \mu \mathrm{l}$ reaction mixture included $2 \mu \mathrm{l}$ of the cDNA product, $12.5 \mu \mathrm{l}$ of SYBR Green Supermix, and $10 \mu \mathrm{M}$ each of the forward and reverse primers. Thermal cycling was performed with the Mx3000P Real-time Quantitative PCR Detection System (Applied Biosystems, Foster City, Calif., USA) under the following conditions: denaturation for $2 \mathrm{~min}$ at $95^{\circ}$, then 40 cycles of denaturation for $30 \mathrm{~s}$ at $95^{\circ}$, annealing for $30 \mathrm{~s}$ at $62.5^{\circ}$ for RIG- 1 and TLR 3 and at $65^{\circ}$ for SOCS 1 and SOCS3, and extension for $30 \mathrm{~s}$ at $72^{\circ}$. The following primers were used: human RIG-I gene forward $5^{\prime}$-GTGGAATCAC GGATTAGC- $3^{\prime}$ and reverse $5^{\prime}$-TTGTCTGGCATCTGGAAC- $3^{\prime}$; human TLR3 gene forward $5^{\prime}$-TTGCTCATTCTCCCTTACAC- $3^{\prime}$ and reverse $5^{\prime}$-CTTCGGAGCATCAGTCGT- $3^{\prime}$; human SOCS1 gene forward $5^{\prime}$-TTGGAGGGAGCGGATGGGTGTAG- $3^{\prime}$ and reverse $5^{\prime}$-AGAGGTAGGAGGTGCGAGTTCAGGTC-3'; human SOCS3 gene forward $5^{\prime}$-ATACTATACCTTCCTGTACCTGGGT

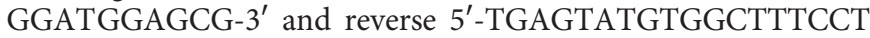
ATGCTGGGTCCCTCT-3'; RSV NS1 gene forward $5^{\prime}$-ATTTA ACTCCCTTGGTTAGAG- $3^{\prime}$ and reverse $5^{\prime}$-GTGTTAGTGACA TTGATTTGC-3', and human glyceraldehyde-3-phosphate dehydrogenase (GAPDH) gene forward $5^{\prime}$-TGATGACATCAAGAAG GTGG-3' and reverse 5' ${ }^{\prime}$-TTACTCCTTGGAGGCCTAGT- ${ }^{\prime}$. The standard curve method and the $\Delta / \Delta \mathrm{Ct}$ method were used to analyze the real-time PCR data. The relative amount of target mRNA $\left(2^{-\Delta \Delta} \mathrm{CT}\right)$ was calculated by normalizing it to the endogenous GAPDH reference.

\section{Immunoblotting}

Cell protein extracts were prepared by adding to the cells a buffer containing $20 \mathrm{mM}$ Tris- $\mathrm{HCl}$ ( $\mathrm{pH} 7.4$ ), 0.5\% sodium deoxycholate, $10 \%$ glycerol, $150 \mathrm{mM} \mathrm{NaCl}, 2 \mathrm{mM}$ EDTA, $50 \mathrm{~mm}$ $\beta$-glycerophosphate, $2 \mathrm{mM} \mathrm{Na}_{3} \mathrm{VO}_{4}, 10 \mathrm{~mm} \mathrm{NaF}, 1 \mathrm{~mm}$ DTT, $1 \mathrm{~mm}$ phenylmethylsulfonyl fluoride, and $0.1 \%$ protease inhibitor cocktail (Roche, Penzberg, Germany). A BCA protein assay kit was used to determine the protein concentrations (Beyotime Institute of Biotechnology, Shanghai, China). The samples were separated by electrophoresis on a $5 \%$ stacking/ $15 \%$ separating sodium dodecyl sulfate polyacrylamide gel and transferred to polyvinylidene difluoride transfer membranes (Immobilon; Millipore, Schwalbach, Germany). The membranes were blocked in TBS-Tween containing $5 \%$ (wt/vol) dry skim milk for $2 \mathrm{~h}$ and then incubated overnight at $4^{\circ}$ with the primary antibodies: rabbit anti-human STAT2 pAb (1:1,000), rabbit anti-human phosphorylated STAT2 (1:500), rabbit anti-human SOCS1 pAb (1:1,000), anti-flag Ab $(1: 1,000)$ and rabbit anti-human GAPDH Ab (Cell Signaling Technology Inc., Mass., USA). The membranes were then incubated for $2 \mathrm{~h}$ with peroxidase-conjugated goat anti-rabbit secondary $\mathrm{Ab}$ $(1: 2,000)$. Antibodies reactive to the protein bands were detected with enhanced chemiluminescence (ECL; Beyotime Institute of Biotechnology, Shanghai, China) and documented with an Image Reader LAS-3000 (Fuji Film, Japan).

\section{Results}

RSV NS1 Upregulates SOCS1 Expression in A549 Cells

To ascertain the regulatory effects of RSV NS1 on the expression of SOCS1 and SOCS3, A549 cells were transfected with pNS1 and real-time PCR was performed to measure the SOCS1 and SOCS3 mRNA levels in a timedependent manner. Cells transfected with the empty vector or infected with RSV were used as the controls. RSV elevated SOCS1 and SOCS3 mRNA expression in the early phase of infection compared with their expression in uninfected cells. The peaks of expression occurred 1-2 $\mathrm{h}$ after infection, and this increase continued for up to $24 \mathrm{~h}$ ( $p<0.05$; fig. 1a). This result is consistent with those of previous studies in which RSV upregulated SOCS1 and SOCS3 mRNA expression in human lung epithelial cells or macrophage-like U937 cells $[11,12]$. By contrast, when the cells were transfected with pNS1, SOCS1 mRNA expression increased 30-fold compared with the baseline level, which was much higher than its expression in the vector-treated cells or in the RSV-infected cells $(\mathrm{p}<0.01)$. Unlike RSV infection, pNS1 expression did not upregulate the expression of SOCS3 mRNA $(p>0.05)$. The reason why pNS1 obtained a higher SOCS1 mRNA levels than RSV infection could be attributed to more NS1 transcripts in plasmids transfection cells.

To further determine whether NS1 is required to induce SOCS1 during RSV infection, an siRNA targeted at NS1 mRNA (siNS1) was used to inhibit the expression of NS1. To evaluate the inhibitory efficacy of the siRNA, A549 cells were treated with siNS1 for $24 \mathrm{~h}$ and then infected with RSV for another $4 \mathrm{~h}$. The cellular mRNA was extracted for real-time PCR analysis. As shown in figure $1 b$, compared with cells treated with the mismatched siRNA (siControl), SOCS1 mRNA expression induced by RSV infection was inhibited in cells pretreated with siNS1 $(\mathrm{p}<0.01)$. These results suggest that the expression of SOCS1 was suppressed because the expression of the NS1 gene was silenced, and that NS1 is an important subunit protein expressed by the RSV genome that induces the expression of SOCS1.

NS1 Induces SOCS1 Independent from RIG-I or TLR3 Pathways

SOCS1 expression has been shown to be upregulated by endocrine type I IFN, induced through the TLR3 and RIG-I signaling pathways [21, 22], while pNS1 transfection also induced RIG-I expression, which implies that endocrine IFN signaling activation could mediate SOCS1 expression. To determine whether NS1 induces SOCS1 via the RIG-I or TLR3 pathway, RIG-I and TLR3 mRNA expression was assessed in cells treated with RSV or pNS1. Figure 1a shows that both RSV and pNS1 induced RIG-I mRNA expression, but RSV infection induced greater expression of RIG-1 mRNA (32-fold increase) and in an earlier phase than did transfection with $\mathrm{pNS} 1(\mathrm{p}<0.01)$. RSV 


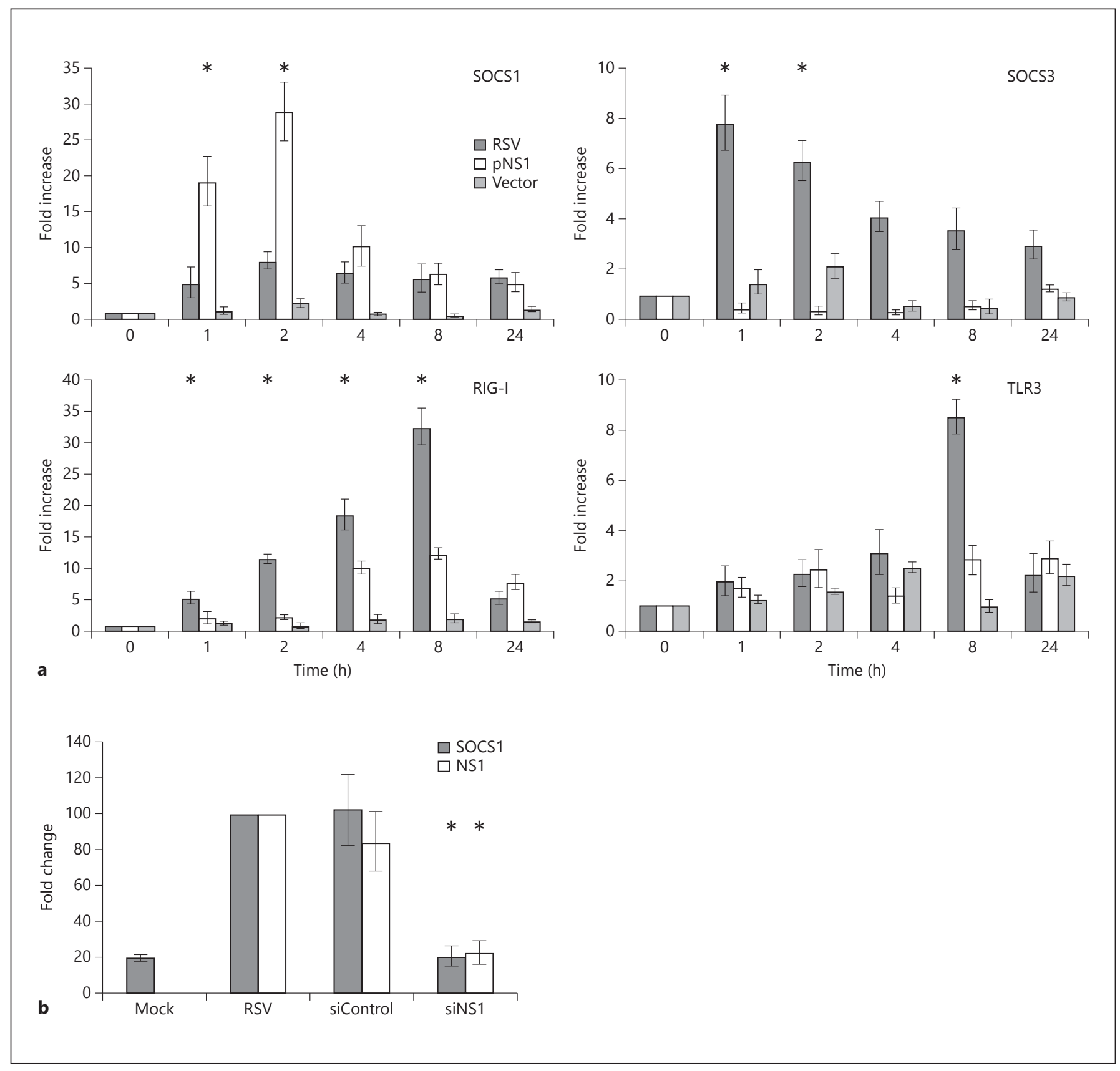

Fig. 1. pNS1 induced SOCS1 mRNA expression in A549 cells. a A549 cells were infected with RSV at a multiplicity of infection (moi) of 3, or transfected with $1.6 \mu \mathrm{g}$ of pNS1 or empty vector in 6 -well plates for $1,2,4,8$, or $24 \mathrm{~h}$. Total cellular RNA was extracted at the indicated times, and real-time PCR was performed to determine SOCS1, SOCS3, RIG-I, and TLR3 mRNA levels. The figure shows the fold increases in mRNA levels compared with the baseline level $(0 \mathrm{~h})$. The data were normalized to the GAPDH mRNA levels and are shown as the means \pm SE of three independent experiments. ${ }^{*} \mathrm{p}<0.01$, pNS1 transfection versus RSV infection. $\mathbf{b}$ siRNA directed against NS1 mRNA in- hibited virus-induced SOCS1 mRNA expression. A549 cells were transfected with siRNA at a final concentration of $40 \mathrm{nM}$ for $24 \mathrm{~h}$ and then infected with RSV at moi $=3$ for $4 \mathrm{~h}$. Total cellular RNA was extracted to determine the NS1 and SOCS1 mRNA levels by reverse transcription and real-time PCR. The figure shows the fold increases in mRNA levels compared with those in RSV-infected cells. The data were normalized to the GAPDH mRNA levels and are shown as the means \pm SE of three independent experiments. ${ }^{*} \mathrm{p}<0.01$, versus the siControl-treated cells. 


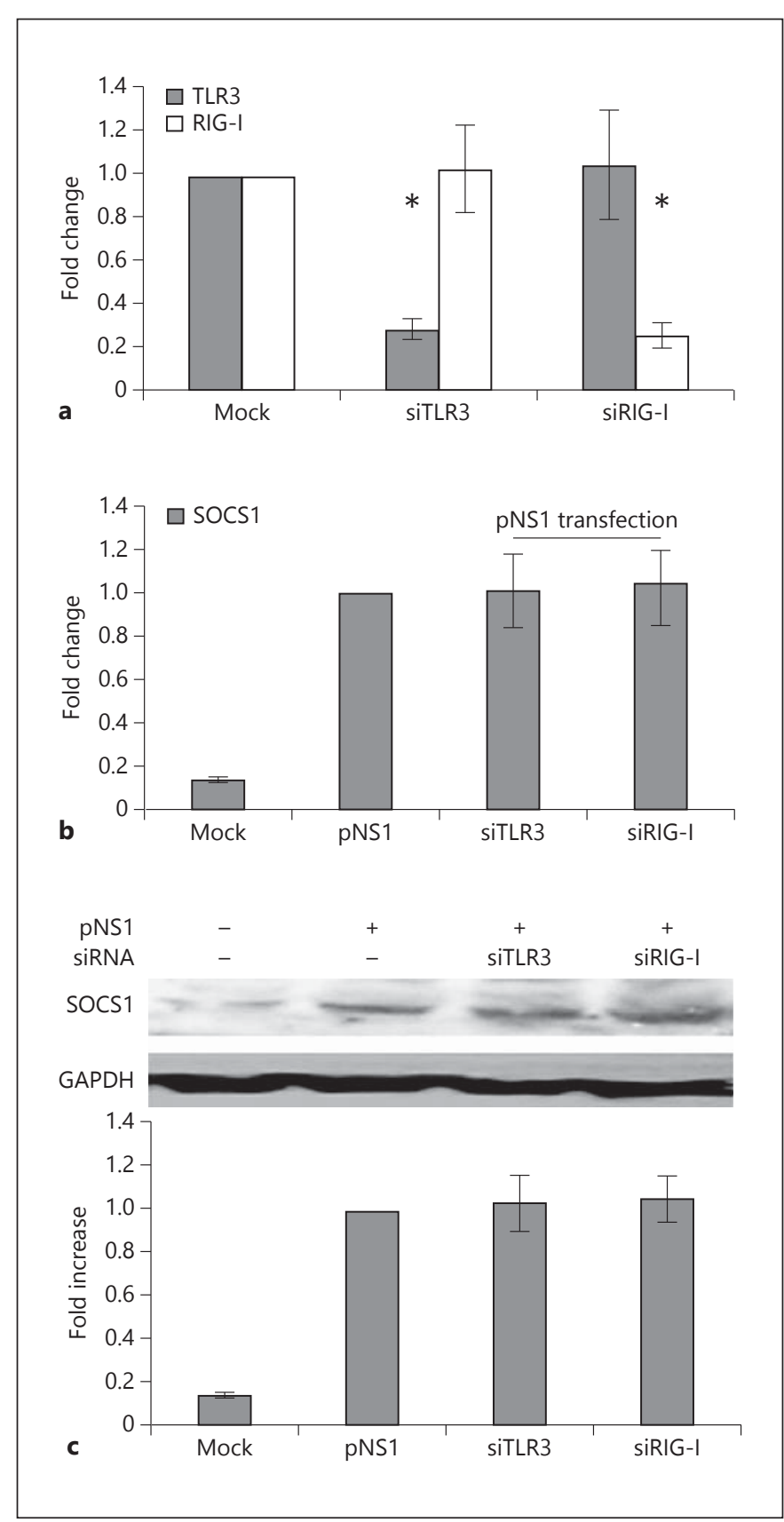

Fig. 2. RSV NS1 upregulated SOCS1 independent from RIG-I or TLR3 pathways. A549 cells were treated with siRNA against to RIG-I or TLR 3 at final concentration of $40 \mathrm{nM}$ for $24 \mathrm{~h}$ and then total cellular RSV was extracted to determine mRNA levels inhibition efficacy using real-time PCR (a). Cells were treated with siRNA against target genes for $24 \mathrm{~h}$ and then transfected with $1.6 \mu \mathrm{g}$ of $\mathrm{pNS} 1$ or the control vectors (mock) for another $4 \mathrm{~h}$. Total cellular RNA was extracted to determine the mRNA levels using real-time PCR (b), and proteins were collected to perform Western blotting (c). The data were normalized to the GAPDH (mRNA or protein) levels and are shown as the means \pm SE of three independent experiments. ${ }^{*} \mathrm{p}<0.01$, versus the Controls.

RSV NS1 Induces SOCS1 to Degrade STAT2 infection induced TLR3 expression, which peaked at $8 \mathrm{~h}$ and declined to normal levels within $24 \mathrm{~h}$, whereas pNS1 had no effect on TLR3 expression. Considering both SOCS1 and RIG-I mRNAs were upregulated under the pNS1 transfection, while SOCS1 mRNA expression is earlier than RIG-I, it could be speculated that SOCS1 expression might be independent of RIG-I pathway. To clarify that, an siRNA method was performed to silence RIG-I or TLR3 mRNA. Unexpectedly, the pNS1-induced SOCS1 mRNA and protein levels were not affected by the genes silence either by RIG-I or TLR3 (fig. 2a-c). These results show that although pNS1 induced the expression of both RIG-I and SOCS1 mRNAs soon after its transfection, SOCS1 mRNA expression was unrelated to either RIG-I or TLR3 signaling activation. Therefore, we conclude that RSV NS1 directly upregulates the expression of SOCS1 mRNA via a RIG-I- and TLR3-independent pathway.

\section{RSV NS1 Inhibits STAT2 and pSTAT2 Expression in Epithelial Cells}

It has been shown that NS1 blocks the IFN-mediated antiviral response by reducing STAT2 levels during RSV infection [6], whereas SOCS1 inhibits STAT2 phosphorylation. However, it is still unclear whether the action of NS1 on STAT2 is independent of SOCS1. To clarify the mechanism involved, A549 cells were treated with IFN- $\alpha$ and then transfected with pNS1, and the cellular proteins were detected by Western blotting analysis at specific time points. NS1 protein was detected as early as $1 \mathrm{~h}$ after the cells were transfected with the expression plasmid (fig. 3a), and NS1 expression inhibited IFN- $\alpha$-induced STAT2 phosphorylation sharply at $4 \mathrm{~h}$ posttransfection and degraded STAT2 in a time-dependent manner compared with those in cells treated with the empty control vector $(p<0.05)$. These results suggest that the RSV NS1 protein impairs type I IFN signal transduction by suppressing the expression of STAT2 and its phosphorylation in A549 cells.

Our previous studies showed that RSV infection upregulates SOCS1 expression, mediated by type I IFN, to inhibit STAT2 phosphorylation [11]. In this study, we examined whether the expression of SOCS1 protein is responsible for the degradation of STAT2 and the reduction of its phosphorylation by NS1. To further assess the functional role of SOCS1 in the NS1-induced suppression of STAT2 phosphorylation, siSOCS1 was used to silence the SOCS1 gene. A549 cells were treated with siSOCS1 or the control siRNA for $24 \mathrm{~h}$ and then transfected with pNS1 for the indicated times. The cellular proteins were extracted and an immunoblot analysis was performed to 


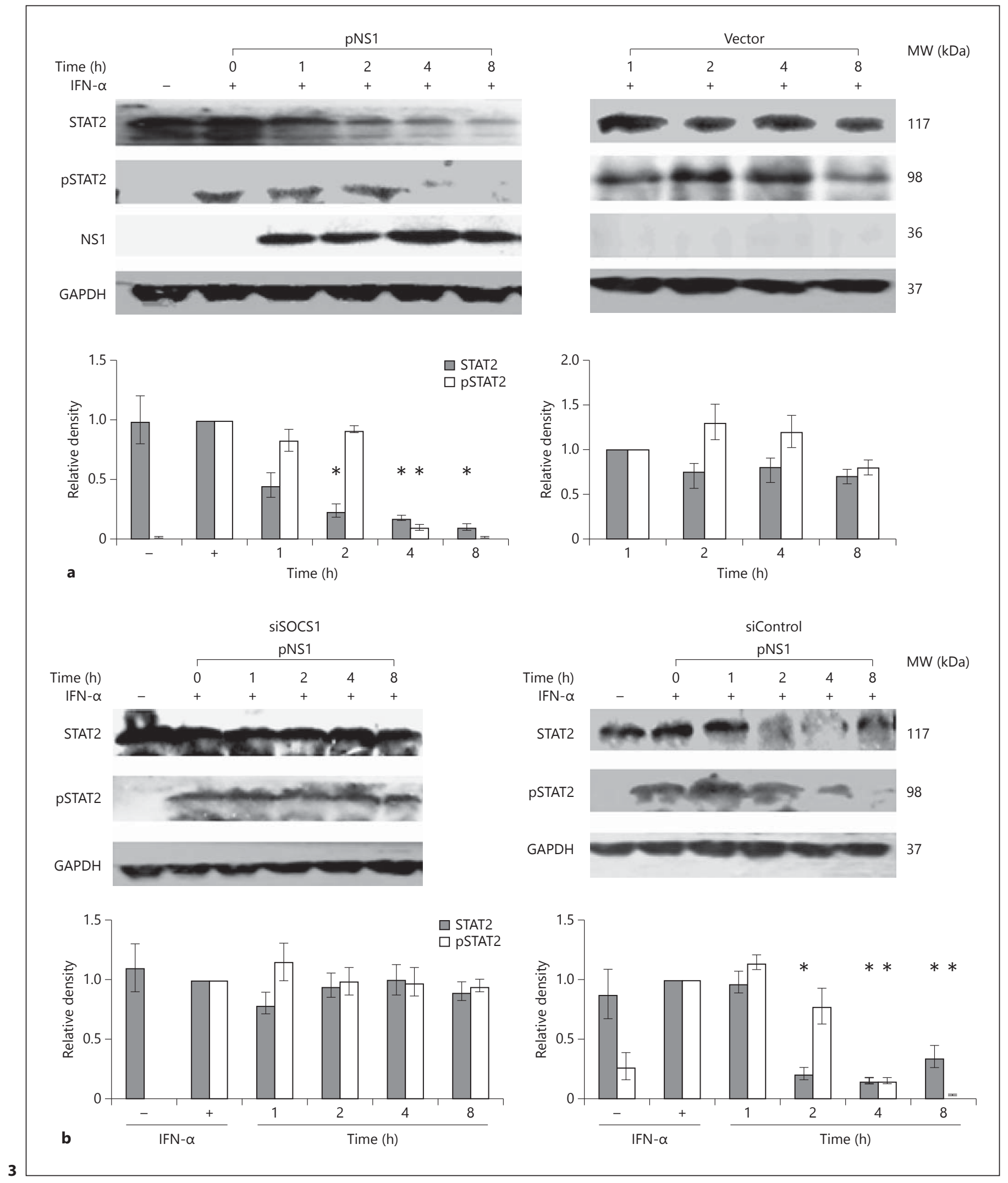

(For legend see next page.) 


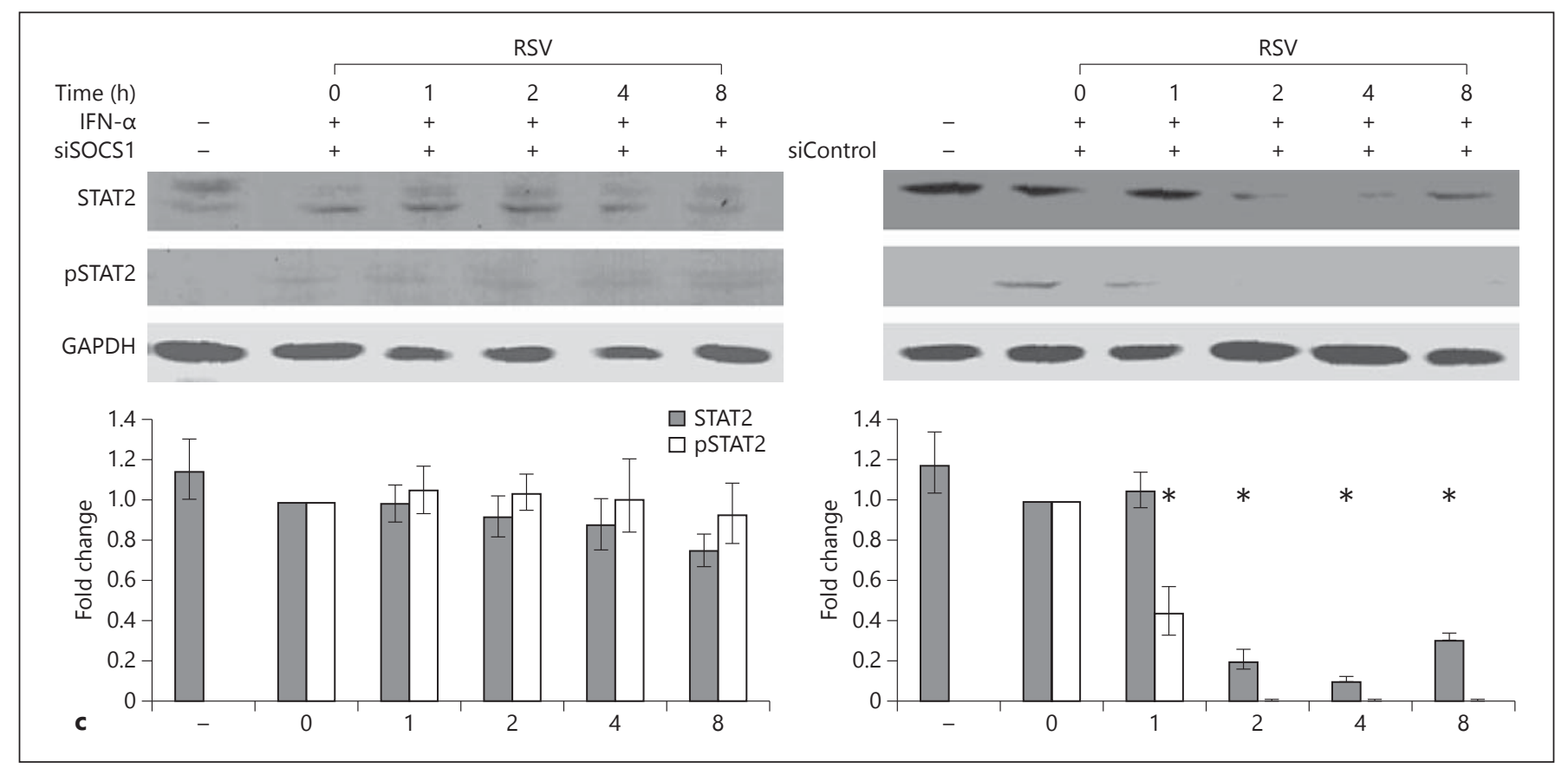

Fig. 3. RSV NS1 inhibited epithelial cell STAT2 and pSTAT2 expression (a). A549 cells were incubated with or without IFN-a (final concentration $1,000 \mathrm{U} / \mathrm{ml}$ ) for $30 \mathrm{~min}$ and then transfected with $4.5 \mu \mathrm{g}$ of empty pcDNA3.1 vector or pNS1 in 6-well plates for the indicated times. The cellular proteins were extracted for Western blotting analysis. STAT2, pSTAT2, and NS1 protein levels were normalized against the GAPDH levels. The figures shown are the relative intensities compared with those for cells incubated with IFN-a without plasmid transfection or cells transfected with empty vector for $1 \mathrm{~h}$. The data shown are the means \pm SE of three independent experiments. ${ }^{*} \mathrm{p}<0.05$, versus time zero. NS1 inhibition of STAT2 phosphorylation is dependent on SOCS1 upregula-

determine the STAT2 expression and phosphorylation induced by IFN- $\alpha$. In the siRNA-control-treated cells, the NS1 expressed from $\mathrm{pNS} 1$ degraded STAT2 $2 \mathrm{~h}$ after plasmid transfection and inhibited STAT2 phosphorylation $4 \mathrm{~h}$ after plasmid transfection $(\mathrm{p}<0.05)$. By contrast, the NS1 expressed from pNS1 inhibited neither STAT2 expression nor STAT2 phosphorylation in the siSOCS1-silenced cells (fig. 3b). This result was consistent with wildtype RSV infection when SOCS1 was silenced by siRNA, although the wild-type RSV infection suppressed pSTAT2 earlier than pNS1 transfection (fig. 3c), which could be attributed to the synergic effect with other subunit proteins such as NS2. These observations suggest that the inhibition of STAT2 expression and phosphorylation by RSV NS1 protein is mediated by the upregulation of SOCS1 protein expression, independently of the activation of type I IFN signaling. tion (b, c). A549 cells were pretreated with siSOCS1 or siControl at a final concentration $40 \mathrm{nM}$ in 6-well plates for $24 \mathrm{~h}$ and then incubated with or without IFN- $\alpha(1,000 \mathrm{U} / \mathrm{ml})$. The cells were then transfected with $4.5 \mu \mathrm{g}$ of pNS1, or incubated with wild-type RSV at mio $=3(\mathbf{b})$. The cell lysates were harvested at the indicated times for Western blotting analysis. STAT2 and PSTAT2 protein levels were normalized to the GAPDH levels (a). The figures shown are the relative intensities compared with those for cells incubated with IFN- $\alpha$ without plasmid transfection. The data shown are the means \pm SE of three independent experiments. ${ }^{*} \mathrm{p}<0.05$, versus siControl-transfected cells of time zero.

\section{Discussion}

The study reported here explored the mechanism by which RSV NS1 protein subverts the antiviral response of the host. NS1 has been proposed as a target for therapeutic strategies directed against RSV. For example, RSV-infected mice treated with interfering NS1 nanoparticles had markedly lower viral titers and airway inflammation in their lungs than the control mice [5]. Similarly, recombinant deletion mutants of RSV that did not express NS1 were shown to be highly attenuated and immunogenic in chimpanzees [24]. Mutants of RSV singly or doubly deleted for NS1 and NS2 demonstrated that the two proteins exert independent and coordinate inhibitory effects on RSV infection, although NS1 plays a more independent role $[8,24,25]$. These studies suggest that NS1 is a major RSV virulence factor, degrading STAT2 by forming a 
functional E3 ligase act as VHL and SOCS proteins [12]. In the present experiments, we clarified the regulatory role of NS1 in SOCS1 gene expression and the impact of this regulatory effect on the downstream JAK/STAT pathway.

SOCS protein is considered to be regulated by a classic negative feedback loop, by balancing physiological effects of cytokines. The virus evades the innate immune surveillance of the host by inducing the expression of the SOCS gene to inhibit the host's IFN-inducible antiviral response $[26,27]$. For instance, RSV infection increases the expression of SOCS1 and SOCS3 mRNAs in macrophage-like U937 cells or Hep-2 cells, and reduces the phosphorylation of STAT1 and STAT2 [9-11]. Influenza A virus inhibits type I IFN signaling via the NF- $\mathrm{KB}$-dependent induction of SOCS3 expression [28]. Another study has shown that an RSV surface protein, not IFN, directly induces SOCS1 expression, which blocks the consequent downstream signaling pathway in an early stage of infection [22]. Other viruses, such as human hepatitis virus C, induce SOCS3 mRNA expression through the C core protein, which is associated with the inhibition of IFN- $\alpha$ inducible tyrosine phosphorylation and activation in human hepatic cells [27]. Recombinant deletion mutants demonstrated that $\Delta \mathrm{NS} 1 / 2$ RSV caused significantly reduced expression of SOCS1 and greater IFN production in MLE-15 cells [22]. These studies suggest that RSV NS1 plays a key role in upregulating SOCS1 expression to subvert the innate immune response of the host.

In the present study, the expression of NS1 from pNS1 apparently upregulated SOCS1 mRNA, but had little effect on SOCS3 mRNA. By contrast, RSV challenge induced both SOCS1 and SOCS3 expression at an early stage of infection. The NS1 gene is located at the $3^{\prime}$-endpoint in the RSV genome and is the first mRNA to be transcribed when the virus replicates. By inducing SOCS1 mRNA, RSV activates a potent mechanism to inhibit the innate antiviral response before the activation of the endocrine IFN. Thus, the virus can replicate successfully under conditions of antiviral signaling suppression. Viral infection triggers the innate antiviral response in part through activation of RIG-I [29]. Our experiments also confirmed that pNS1 and RSV induce RIG-I mRNA expression. Recently, RSV NS1 has been found to bind MAVS and inhibit the MAVS-RIG-I interaction required for IFN production [30]. This should explain the reason why RSV is a poor IFN inducer although it upregulates RIG-I expression.

To determine whether the effect of NS1 is independent of the IFN pathway, RIG-I and TLR3 mRNAs were also examined. Both NS1 and RSV upregulated RIG-I expression, but NS1 induced less RIG-I mRNA than RSV, and this induction began to increase at $4 \mathrm{~h}$ after the expression of SOCS1 and $1 \mathrm{~h}$ after plasmid transfection. TLR3 mRNA expression showed a nonsignificant increase when the cells were challenged with pNS1. These data imply that RSV NS1 protein upregulates the expression of SOCS1 through a TLR3- and RIG-I-independent pathway. One mechanism that the paramyxoviruses may use to regulate IFN signaling by the host is blocking the JAK/STAT signaling pathway $[8,31]$. Numerous studies have shown that RSV NS1 and NS2 proteins are type I IFN antagonists and target both IFN induction and the activation of IFN signaling. RSV suppresses type I IFN signaling by reducing STAT2 protein, and both NS1 and NS2 have been shown to play roles in this response [5, 7, 8, 13, 24, 28]. In our experiments, NS1 reduced STAT2 protein expression and the STAT2 phosphorylation induced by IFN- $\alpha$. Further studies should demonstrate that the expression of SOCS1 protein is responsible for the reduction by NS1 of STAT2 expression and phosphorylation. These results suggest that RSV NS1 protein inhibits the expression and phosphorylation of STAT2, leading to the impairment of IFN- $\alpha$-inducible antiviral signaling.

In summary, we conclude that RSV NS1 protein upregulates SOCS1 mRNA independently of the TLR3 and RIG-I signaling pathways and that the upregulation of SOCS1 could be responsible for the inhibition of STAT2 activation by NS1. Inhibiting SOCS1 expression might be a therapeutic option to prevent RSV replication and reduce the local inflammatory response in the airways.

\section{Acknowledgement}

This work was supported by China National Natural Science Foundation (Nos. 30973220 and 81170005).

References

${ }_{1}$ Sigurs N, Bjarnason R, Sigurbergsson F, Kjellman B: Respiratory syncytial virus bronchiolitis in infancy is an important risk factor for asthma and allergy at age 7. Am J Respir Crit Care Med 2000;161:1501-1507.

-2 Falsey AR, Hennessey PA, Formica MA, Cox C, Walsh EE: Respiratory syncytial virus infection in elderly and high-risk adults. N Engl J Med 2005;352:1749-1759.

- 3 Schickli JH, Dubovsky F, Tang RS: Challenges in developing a pediatric RSV vaccine. Human Vaccines 2009;5:582-591.

4 Schlender J, Bossert B, Buchholz U, Conzelmann KK: Bovine respiratory syncytial virus nonstructural proteins NS1 and NS2 cooperatively antagonize $\alpha / \beta$ interferon-induced antiviral response. J Virol 2000;74:8234-8242. 
-5 Zhang W, Yang H, Kong X, Mohapatra S, San Juan-Vergara H, Hellermann G, Behera S, Singam R, Lockey RF, Mohapatra SS: Inhibition of respiratory syncytial virus infection with intranasal siRNA nanoparticles targeting the viral NS1 gene. Nat Med 2005;11:56-62.

6 Lo MS, Brazas RM, Holtzman MJ: Respiratory syncytial virus nonstructural proteins NS1 and NS2 mediate inhibition of Stat2 expression and $\alpha / \beta$ interferon responsiveness. J Virol 2005;79:9315-9319.

7 Spann KM, Tran KC, Chi B, Rabin RL, Collins PL: Suppression of the induction of $\alpha, \beta$, and $\gamma$ interferons by the NSI and NS2 proteins of human respiratory syncytial virus in human epithelial cells and macrophages. J Virol 2004; 78:4363-4369.

8 Oshansky CM, Zhang W, Moore E, Tripp RA: The host response and molecular pathogenesis associated with respiratory syncytial virus infection. Future Microbiol 2009; 279-297.

-9 Ramaswamy M, Shi L, Monick MM, Hunninghake GW, Look DC: Specific inhibition of type I interferon signal transduction by respiratory syncytial virus. Am J Respir Cell Mol Biol 2004;30:893-900.

10 Zhao D, Yan T, Li L, You S, Zhang C: Respiratory syncytial virus inhibits interferon- $\alpha$ inducible signaling in macrophage-like U937 cells. J Infect 2007;54:393-398.

11 Hashimoto K, lshibashi K, Ishioka K, Zhao D, Sato M, Ohara S, Abe Y, Kawasaki Y, Sato Y, Yokota S, Fujii N, Peebles RS Jr, Hosoya M, Suzutani T: RSV replication is attenuated by counteracting expression of the suppressor of cytokine signaling (SOCS) molecules. Virology 2009;391:162-170.

12 Elliott J, Lynch O, Suessmuth Y, Qian P, Boyd C, Burrows J, Buick R, Stevenson N, Touzelet O, Gadina M, Power U, Johnston J: Respiratory syncytial virus NS1 protein degrades STAT2 by using the elongin-cullin E3 ligase. J Virol 2007;81:3428-3436.

13 Yoshimura A, Naka T, Kubo M: SOCS proteins, cytokine signalling and immune regulation. Nat Rev Immunol 2007;7:454-465.
14 Kile BT, Schulman BA, Alexander WS, Nicola NA, Martin HM, Hilton DJ: Opening Pandora's SOCS box - a tale of destruction and degradation. Trends Biochem Sci 2002;27: 235-241.

15 O'Shea J, Gadina M, Schreiber R: Cytokine signaling in 2002: new surprises in the Jak/ Stat pathway. Cell 2002;109(suppl):S121S131.

16 Krebs DL, Hilton DJ: SOCS proteins: negative regulators of cytokine signaling. Stem Cells 2001;19:378-387.

17 Akhtar LN, Benveniste EN: Viral exploitation of host SOCS protein functions. J Virol 2011; 85:1912-1921.

18 Kawai T, Akira S: The role of pattern-recognition receptors in innate immunity: update on Toll-like receptors. Nat Immunol 2010;11: 373-384.

19 Le Goffic R, Pothlichet J, Vitour D, Fujita T, Meurs E, Chignard M, Si-Tahar M: Cutting edge: influenza A virus activates TLR3-dependent inflammatory and RIG-I-dependent antiviral responses in human lung epithelial cells. J Immunol 2007;178:3368-3372.

20 Pothlichet J, Chignard M, Si-Tahar M: Innate immune response triggered by influenza A virus is negatively regulated by SOCS1 and SOCS3 through a RIG-I/IFNAR1-dependent pathway. J Immunol 2008;180:2034-2038.

-21 Oshansky CM, Krunkosky TM, Barber J, Jones LP, Tripp RA: Respiratory syncytial virus proteins modulate suppressors of cytokine signaling 1 and 3 and the type I interferon response to infection by a toll-like receptor pathway. Viral Immunol 2009;22: 147-161.

22 Moore EC, Barber J, Tripp RA: Respiratory syncytial virus attachment and nonstructural proteins modify the type I interferon response associated with suppressor of cytokine signaling proteins and IFN-stimulated gene-15. Virol J 2008;5:116.
23 Ramaswamy M, Shi L, Varga SM, Barik S, Behlke MA, Look DC: Respiratory syncytial virus nonstructural protein 2 specifically inhibits type I interferon signal transduction. Virology 2006;344:328-339.

24 Spann KM, Tran KC, Collins PL: Effects of nonstructural proteins NS1 and NS2 of human respiratory syncytial virus on interferon regulatory factor $3, \mathrm{NF}-\mathrm{\kappa B}$, and proinflammatory cytokines. J Virol 2005;79:5353-5362.

25 Swedan S, Andrews J, Majumdar T, Musiyenko A, Barik S: Multiple functional domains and complexes of the two nonstructural proteins of human respiratory syncytial virus contribute to interferon suppression and cellular location. J Virol 2011;85:10090-10100.

26 Brand S, Zitzmann K, Dambacher J, Beigel F, Olszak T, Vlotides G, Eichhorst S, Goke B, Diepolder H, Auernhammer C: SOCS-1 inhibits expression of the antiviral proteins $2,5^{\prime}$ OAS and MxA induced by the novel interferon- $\lambda s$ IL-28A and IL-29. Biochem Biophys Res Commun 2005;331:543-548.

27 Bode JG, Ludwig S, Ehrhardt C, Albrecht U, Erhardt A, Schaper F, Heinrich PC, Häussinger D: IFN- $\alpha$ antagonistic activity of HCV core protein involves induction of suppressor of cytokine signaling-3. FASEB J 2003;17:488-490.

28 Pauli EK, Schmolke M, Wolff T, Viemann D, Roth J, Bode JG, Ludwig S: Influenza A virus inhibits type I IFN signaling via NF- $\mathrm{BB}$ dependent induction of SOCS3 expression. PLoS Pathog 2008;4:e1000196.

29 Moresco EMY, La Vine D, Beutler B: Prionlike behavior of MAVS in RIG-I signaling. Cell Res 2011;21:1643-1645.

-30 Boyapalle S, Wong T, Garay J, Teng M, San Juan-Vergara H, Mohapatra S, Mohapatra S: Respiratory syncytial virus NS1 protein colocalizes with mitochondrial antiviral signaling protein MAVS following infection. PloS One 2012; 7:e29386

- 31 Bousse T, Chambers RL, Scroggs RA, Portner A, Takimoto T: Human parainfluenza virus type 1 but not Sendai virus replicates in human respiratory cells despite IFN treatment. Virus Res 2006;121:23-32. 